\title{
亶 \\ O rúmen como marcador de saúde: revisão de literatura
}

[The rumen as a health thermometer: literature review]

\section{"Revisão/Review"}

\section{Bruna de Souza Silva Higino ${ }^{*}$, Saulo Gusmão Silva De Tarso²}

\author{
${ }^{1}$ Programa de Pós-Graduação em Medicina Veterinária, Departamento de Medicina Veterinária, Universidade Federal \\ Rural de Pernambuco, Recife- PE, Brasil. \\ ${ }^{2}$ Programa de Pós-Graduação em Sanidade e Reprodução de Ruminantes, Unidade Acadêmica de Garanhuns, \\ Universidade Federal Rural de Pernambuco, Garanhuns-PE, Brasil. \\ *Autor para correspondência/Corresponding author: E-mail: brunhssilva@ hotmail.com
}

\section{Resumo}

A avaliação ruminal permite a observação do funcionamento do organismo dos ruminantes como um todo, e tem se destacado a medida que a procura por proteína animal avança. Além disso, as maiores exigências de mercado e do produtor em busca de resultados com um menor tempo, são fatores que justificam os estudos em espécies ruminantes. O compartimento fermentativo do sistema digestório desses animais demonstra modificações a nível sistêmico, inclusive na identificação de predisposição de doenças. Devido à crescente mudança dietética em busca da eficiência na produção, fez-se necessário o aprofundamento sobre as possíveis consequências. O ecossistema complexo do rúmen necessita de aspectos próprios para seu bom desempenho. A fisiologia ruminal diverge entre as espécies e os próprios indivíduos, além de ser influenciado diretamente pelas inúmeras dietas a que podem ser submetidos, sendo o rúmen um órgão capaz de indicar o estado metabólico do animal. O uso de parâmetros de comportamento ruminal como marcadores da saúde de rebanhos, aparece como uma ferramenta inovadora e promissora diante dos desafios atuais na produção de ruminantes.
\end{abstract}

Palavras-chave: poligástricos; doenças metabólicas; produção animal; rebanho.

\begin{abstract}
The ruminal evaluation permits observation of the functioning of the ruminant's body as a whole and has been a novel tool to improve animal production. The fermentative compartment of the digestive system of these animals demonstrates changes that could be used as a tool for identification of predisposition to disease. The complex rumen ecosystem needs proper aspects for its perfect performance. Also, because of the direct influence of the numerous diets that can be injested, the rumen stands as an organ capable of indicating the metabolic state of the animal. Therefore, the use of ruminal behavior parameters as markers of herd health appears as a novel and promising tool in the face of current challenges in ruminant production.
\end{abstract}

Keywords: polygastric; metabolic diseases; animal production; flock.

\section{Introdução}

A pecuária passou por fortes modificações e o crescente avanço na economia mundial do agronegócio ampliou a produção e o progresso das tecnologias voltados a essa área. O Brasil junto aos Estados Unidos são líderes em exportação de proteína animal (Ximenes, 2018), cooperando no valor da manufaturação da economia pecuária (FAO/AIEA, 2005). Porém, grandes desafios têm sido direcionados no que se refere ao alcance à demanda de produtos de origem animal projetada para o futuro. Estima-se que será necessário mais que a duplicação da produção pecuária atual, até 2050 (Alexandratos e Bruinsma, 2012).

A particularidade do sistema digestório dos ruminantes (multicavitário), e a capacidade de digestão fermentativa pré-gástrica do rúmen, faz este tipo de herbívoro alimentar-se de forma mais eficiente da celulose, e especialmente permitindo o melhor aproveitamento de recursos forrageiros de qualidade baixa. Mudanças e adequações 
morfológicas aconteceram evolutivamente ao longo dos séculos nos ruminantes, fazendo com que estes animais pudessem aumentar por exemplo a capacidade de alimento ingerido e o ganho de massa coeporal através da digestão de alimentos considerados relativamente pobres (De Tarso et al., 2016). Recentemente, o novo cenário de intensificação dos sistemas de produção, aplicando dietas ricas em concentrado por um longo período de tempo, tem provocado grandes impactos na estrutura morfológica do epitélio ruminal $\mathrm{e}$ colônico de caprinos e bovinos, gerando consequências de ordem celular estrutural e genética do ambiente fermentativo destes animais (Steele et al., 2011a; Steele et al., 2011b).

Modificações no manejo alimentar, principalmente de forma abrupta, aumentam a chance de doenças ruminais e/ou metabólicas, o que pode ocasionar danos severos a longo prazo (Clauss et al., 2003). Métodos de determinação do tempo de ruminação podem presumir o bem estar dos rebanhos e detectar a chance de doenças no futuro através do comportamento ruminal (Calamari et al., 2014; Kaufman et al., 2016).

A ruminação é um ciclo essencial o qual pode ser modificado de modo multifatorial através, por exemplo, de doenças intecorrentes, estresse ou erros de manejo (Welch e Smith, 1970; Beauchemin, 1991; Albright, 1993). Estes fatores são capazes de ocasionar variações metabólicas como consequência, que podem levar a diminuição do fluxo da corrente sanguínea que alimenta o epitélio ruminal e a redução da taxa de digestão (Soriani et al., 2013).

Dietas comfibra efetiva intensificam o processo de ruminação e estimulam a produção de saliva, que favorece o tamponamento, a degradação do conteúdo ruminal, e que preserva ideais condições para sintetização de bactérias celulolíticas, diminuindo flutuações no $\mathrm{pH}$ do rúmen, mantendo o turnover ruminal $\mathrm{e}$ a reabsorção do nitrogênio pelo ciclo da ureia (Calamari et al., 2014). Mesmo sendo passível de variações, a ruminação acontece por cerca de oito horas ao dia, fracionado, com episódios de aproximadamente cinquenta segundos de duração para gerar um ciclo completo (Van Soest, 1994).

A busca por esse entendimento sobre o comportamento ruminal tem ganhado destaque devido as associações com a sanidade em sistemas intensivos modernos. Assim, métodos preventivos e de identificação de animais doentes no rebanho têm sido desenvolvidos na busca por biomarcadores de doenças (Weary et al., 2009). A indicação de que o desempenho da ruminação pode ser um indicador promissor das condições de saúde, criou uma associação entre o tempo de ruminação e condições metabolicas, capaz de avaliar o desempenho dos rebanhos focado no comportamento alimentar (Soriani et al., 2012; De Tarso, 2017).

A diminuição da dinâmica ruminal deve ser considerada um sinal objetivo e importante demonstrado em qualquer situação de anormalidade. $\mathrm{O}$ monitoramento da ruminação passa a ser uma medida essencial por demonstrar modificações de extrema importância que ocorrem no rúmen e que acarretará na diminuição da produção (Soriani et al., 2012). Desta forma, tornase relevante aprofundar estudos sobre a maneira que essa mudança afeta e sua associação com doenças metabólicas.

\section{Variáveis que influenciam no processo de ruminação}

O rúmen dispõe de aspectos próprios para o seu bom funcionamento, o que o torna um ambiente anaeróbico favorável ao crescimento da microbiota. A temperatura ruminal varia entre $38^{\circ} \mathrm{C}$ e $42^{\circ} \mathrm{C}$ conservada através do organismo por meios homeotérmicos. $\mathrm{O} \mathrm{pH}$ em condições fisiológicas é variável entre 6,6 e 7,4, de acordo com a duração de exposição, regularidade de fornecimento e dieta consumida. A quantidade de saliva produzida de forma excessiva pode prejudicar a capacidade tampão da câmara fermentativa, assim como a presença de oxigênio e água. A microbiota ruminal é demasiadamente complexa e pode ser alterada por diversos fatores intra ou extra ruminais (Van Soest, 1994).

O centro gástrico sofre estimulação por meio da tensão e mastigação, e modificações são capazes de alterar a motilidade ruminoreticular. Febre, dor, distensão ruminal e acréscimo na aglomeração dos ácidos graxos voláteis (AGV's) são os principais impulsos dominadores das depressões relacionadas ao centro gástrico. Entretanto, enfermidades ligadas ao abomaso, efeitos colaterais e adversos de medicamentos depressores, hiperglicemia e controle hormonal também são capazes de gerar a redução da motilidade do rúmen-retículo (Constable et al., 1990; De Boever et al., 1990).

A dor tem relação direta com a diminuição da motilidade da câmara fermentativa e interfere através da ativação do nervo esplênico motor pelo Sistema Nervoso Simpático. As prostaglandinas 
são responsáveis por essa expansão dolorosa tanto de forma focal quanto generalizada e seus mecanismos ainda não são totalmente esclarecidos (Constable et al., 1990; Almeida et al., 2008).

Acrescenta-se também que a atonia ruminal está associada à endotoxemia recorrente, febre e anorexia, que acontece devido a um mecanismo associado à prostaglandina que atenua-se pela administração de anti-inflamatórios não esteroides. Drogas anestésicas, no geral que atuam no Sistema Nervoso Central, são depressoras do movimento reticuloruminal, a ação desse sistema se amplia quando há presença de endotoxinas na corrente sanguínea e atuação de receptores $\alpha$-2 adrenérgicos devido a ligação direta com o centro gástrico. A ingestão de alimentos com baixos teores de fibra e ricos em água, promovem uma redução na tensão muscular reticuloruminal que interfere negativamente na dinâmica dos mesmos. (De Boever et al., 1990; Almeida et al., 2008).

A distensão do rúmen desempenha uma consequência inibitória na taxa de passagem ruminal, que ocorre possivelmente devido o acionamento constante dos receptores epiteliais, situados nos pilares ruminais e papilas reticulares no saco cranial de alto limiar mecânico. A indigestão vagal se encaixa como um exemplo importante desta inibição, nestes casos, a lesão nervosa causa retenção de conteúdo no interior do rúmen junto a atonia do órgão (Constable et al., 1990; Van Soest, 1994).

Da mesma forma, o $\mathrm{pH}$ ruminal abaixo de 5 implica diretamente na concentração de ácidos graxos voláteis (AGVs), que interferem no funcionamento do rúmen quando são diluídos em meio ao fluído ruminal, sendo identificados pelos receptores epiteliais que bloqueiam impulsos para o centro gástrico. A acidez causada pelo acúmulo de ácido láctico gera altos coeficientes de AGV's não dissociados que resulta na atonia do rúmen. $\mathrm{A}$ acidez a nível sanguíneo não interfere tão severamente na motilidade, apesar de contribuir com sua redução (Constable et al., 1990; Ametaj et al., 2010a; Plaizier et al., 2012).

É bem estabelecido que o mau funcionamento ruminal acarreta em uma inflamação sistêmica que favorece o aparecimento de distúrbios como laminite, hipocalcemia, retenção de placenta, metrite e cetose (Ametaj et al., 2010a; Plaizier et al., 2012; Bradford et al., 2015).

A privação de água, desequilíbrio eletrolítico e hiperglicemia alteram o mecanismo do rúmen e retículo, assim como a ação das bactérias celulolíticas, pois estas exercem influência no mecanismo ruminal e na entrada e saída de alimentos. Os ruminantes devem ingerir um volume entre 10 e $15 \%$ do peso vivo, de água para assegurar a boa atividade do trato gastrintestinal (Zebeli e Metzler-Zebeli, 2012; Zebeli et al., 2015).

Enfermidades relacionadas ao abomaso, influenciam também na dinâmica dos préestômagos. Distensão, compactação, torção ou deslocamento são fatores de diminuição da motilidade, de forma que os receptores de tensão do abomaso os identificam e refletem hipomotilidade ruminal (Clauss et al., 2003; Stangaferro et al., 2016).

\section{Comportamento ruminal como marcador para identificar animais com risco de doenças}

$\mathrm{O}$ rúmen exerce uma função essencial na digestão, no feedback imunitário e no bem-estar animal (Zebeli e Metzler-Zebeli, 2012). O ecossistema e o epitélio do rúmen são extremamente importantes para a saúde dos ruminantes pela capacidade de protegê-lo de condições desfavoráveis (Chen et al., 2012; Hollmann, 2013). Indícios demonstram que doenças e a predisposição a elas, podem ser percebidas através do comportamento animal em geral, sobretudo o alimentar, o que permite a prevenção de possíveis distúrbios nos rebanhos (Weary et al., 2009).

Hipóteses relacionadas a interferência ruminal na verificação de doenças se fortificam diante de pesquisas, nas quais relatam que fêmeas bovinas com infecções uterinas tiveram diminuição na ingestão alimentar (Huzzey et al., 2007), entre outras evidências, como a redução da ruminação durante o período pré e pós-parto. A avaliação ruminal, especificamente, pode e deve ser utilizada como um importante identificador de sanidade do rebanho (Calamari et al., 2014).

Tecnologias como a aferição da temperatura retículoruminal, possibilitam de forma eficiente a constatação do estro e do parto em vacas, sendo um sistema de uso intraruminal interessante por não ser invasivo (Cooper-Prado et al., 2011; Suthar et al., 2012). Esta técnica permite uma menor utilização de mão de obra e diminuição das chances de perda do aparelho de aferição (Sievers et al., 2004; Burfeind et al., 2011).

Desta forma, pode-se verificar que perturbações ruminais são multifatoriais 
prejudicando a digestão, o metabolismo, a saúde, a performance e a produtividade em vacas, tornando o monitoramento da saúde do comportamento alimentar essencial para um bom desempenho dos animals no rebanho (Zebeli et al., 2015).

\section{Comportamento ruminal, estado metabólico e associações com doenças}

No momento em que a simbiose do ecossistema ruminal sofre alteração, ocorre uma instabilidade nos componentes da corrente sanguínea provinda de seus mecanismos homeostáticos (Ametaj et al., 2010b; Bradford et al., 2015).

A resposta imunológica inata se ativa através de elevados níveis de citocinas e proteínas de fase aguda, que caracterizam processos inflamatórios que são intimamente relacionados a distúrbios ruminais. $\mathrm{O}$ desequilíbrio dos microrganismos ruminais provoca uma série de modificações no metabolismo do rúmen, o que causa liberação de toxinas por todo o sistema digestório, que se associam a instabilidade sistêmica (Ametaj et al., 2010b; Plaizier et al., 2012; Zebeli e MetzlerZebeli, 2012).

A inconstância ruminal passou a ser uma das causas de inflamação sistêmica, principalmente em vacas que passam pelo período de transição (Catalani et al., 2010; Graugnard et al., 2013; Gressner et al., 2013), o que amplia as chances do animal adquirir afecções digestórias e locomotoras como acidose ruminal, deslocamento de abomaso, timpanismo e laminite (Almeida et al., 2008; Bradford et al., 2015).

Essas enfermidades ainda se associam a baixa ingestão de matéria seca, o que expande o balanço energético negativo (BEN). Em vacas, o BEN durante o período de lactação demonstrou retardar a re-estruturação do útero assim como o desempenho da atividade ovariana e sinais de estro (Butler e Smith, 1989). A gravidade desse fenômeno pode ser medida e avaliada, mesmo que de forma indireta, pelo escore corporal (Schröder e Staufenbiel, 2006).

A alta exigência energética de uma prenhez em confronto com a diminuição do espaço físico da fêmea bovina, juntamente com a abrupta produção de colostro e leite são fatores determinantes a ocorrência de doenças (Lacetera et al., 2005; Ospina et al., 2010; Esposito et al., 2014). Além disso, há uma abrangência no desenvolvimento da relação do metabolismo de energia e gordura associados a inflamação
(LeBlanc, 2012). Muitas vezes, vacas de aptidão leiteira possuem uma dieta mais energética durante o começo da lactação, a qual sendo brusca, resulta na liberação de endotoxina, provinda do rúmen, na circulação (Khafipour et al., 2009).

Em vacas durante o período de transição, a inflamação sistêmica pode causar o surgimento de lipidose no fígado, o que danifica o metabolismo não apenas no fígado. Há uma hipótese de que essa aglomeração de lipídios no fígado pode ser capaz de diminuir a aptidão dos hepatócitos que interfere nos hormônios envolvidos na reprodução. A esteatose hepática diminui a resposta da somatotropina bovina recombinante (BST) reduzindo os níveis circulantes do fator de crescimento semelhante a insulina tipo 1 (IGF-1), que por sua vez age simultaneamente com as gonadotrofinas (LH e FSH) para o estímulo do crescimento folicular (Oliveira, 2004; Bradford, 2009; Reynolds et al., 2003).Dessa forma, entendese que além do funcionamento da digestão, a modificação a nível ruminal afeta o estado metabólico, a sanidade do animal no geral e, principalmente a fertilidade do rebanho. A atenção à saúde do rúmen gera uma taxa de reprodução com maior eficiência, principalmente durante o período de sensíveis transformações de manejo, carga energética e carga hormonal, como o período de transição (Schröder e Staufenbiel, 2006).

\section{Tempo de ruminação como um indicador de bem-estar dos rebanhos}

$\mathrm{O}$ ato de pastar dos ruminantes, sua ingestão de água, ócio, defecação, micção, locomoção e ruminação são essenciais para o bem-estar. A microbiota ruminal e seu funcionamento refletem a alimentação, a idade, as condições geográficas e a saúde do animal (Pers-Kamczyc et al., 2011).

O tempo de ruminação (TR) é um indicativo utilizado como um meio de monitoração de sanidade, estado nutricional e conforto, usado por exemplo com maior frequência em rebanhos leiteiros para prognosticar afecções metabólicas e precisar o momento do parto (De Boever et al., 1990; Soriani et al., 2012; Schirmann et al., 2013; Soriani et al., 2013). Mesmo com as características individuais de cada ruminante, o tempo de ruminação age como um dado informativo adicional eficiente (De Boever et al., 1990) e a tecnologia permite esse acompanhamento através de monitores de som e de vibração (Watanabe et al., 2008). Estudos também relatam a importância da coleta de informações do rebanho, do indivíduo, 
e cita o tempo de ruminação como uma das informações mais relevantes (Urton et al., 2005).

Leves modificações no funcionamento ruminal são habilitadas para fornecer informações inclusive de distúrbios subclínicos, o que permite ações profiláticas. As inflamações uterinas, diante o acompanhamento ruminal, podem ser previstas até duas semanas antes da manifestação clínica (Huzzey et al., 2007). Além disso, a redução do TR e o aumento da atividade diária do animal podem demonstrar fêmeas bovinas no estro e são possíveis detectores de cio silencioso (Reith et al., 2014). De acordo com Kovács et al. (2017), a redução da temperatura retículo-ruminal quando comparada a basal pode prever o momento do parto e auxiliar em vacas com histórico de distorcias. Ademais, a determinação da rumi-

nação pode prover dados de mudanças de $\mathrm{pH}$ e fermentação ruminal. Avaliam também, que a motilidade ruminal amplia a absorção dos ácidos graxos de cadeia curta, consequentemente favorecem o equilíbrio ácido-básico do conteúdo ruminal (Allen, 1997).

Com base em uma diversidade de pesquisas, a monitoração da ruminação vem a abranger e coletar informações cada vez mais amplas, o que destaca sua importância na detecção de distúrbios metabólicos, digestivos e também reprodutivos (Stangaferro et al., 2016).

\section{Comportamento ruminal em diferentes espécies de ruminantes}

A conduta alimentar compreende a dieta, a seleção e ingestão de alimentos, e a ruminação, condição influenciada pelo sistema de criação. A dieta do animal segue como o fator influenciador de maior importância para o funcionamento ruminal. As caraterísticas físicas e químicas dos alimentos podem modificar e determinar o modo de deterioração e a eficácia ruminal, como exemplo tem-se o volume e tamanho das partículas que interferem diretamente na taxa de passagem do rúmen, a presença, população e crescimento microbiano (Martz e Belyea, 1986).

Diferentes espécies de ruminantes possuem a degradação, atividade enzimática e a fermentação ruminal distintas por seus hábitos e artifícios alimentares desiguais, além de variações anatômicas e fisiológicas. Pequenos ruminantes têm um rúmen menor, omaso mais desenvolvido e intestino grosso maior se comparado a ruminantes de grande porte, o que faz com que a permanência de conteúdo ruminal seja reduzida e a taxa de passagem seja maior em ovinos e caprinos (Van Soest, 1994; De Tarso et al., 2016).

Devido a taxa de permanência das partículas alimentares na câmara fermentativa, as cabras possuem uma frequência alimentar e ruminação um pouco distintas dos bovinos. Outras pesquisas afirmam que o número de bactérias ruminais totais de ovinos é superior aos bubalinos (Prasad e Pradhan, 1990). Os bubalinos diferentemente dos bovinos, têm maior atividade de bactérias degradadoras de celulose, consequentemente um proveito maior de dietas fibrosas e uma quantidade maior de protozoários, além do $\mathrm{pH}$ mais básico devido a maior salivação (Tewatia e Bhatia, 1998).

O comportamento alimentar dos ruminantes pode também ser interferido por fatores como idade, peso, estado físiológico e nível de exigência de produção, porém modificações de maior ênfase são ressaltadas na conduta alimentar entre ruminantes da própria espécie se confrontados com outras espécies (Albright, 1993).

\section{O efeito da intensificação dos sistemas no ambiente ruminal}

A modernização dos mercados e a globalização fez com que a busca pela produção animal fosse otimizada. Modificações da dieta passaram a ser o método mais eficiente de atingir eficiência de produção, porém com maior consequência aos processos fermentativos, obrigando adaptações ruminais mais desafiadoras (De Tarso et al., 2016).

Inovações tecnológicas são necessárias para acompanhar os métodos alimentares modernos, como exemplo, o processamento de grãos e seus procedimentos mecânico-térmicos, que buscam a diminuição dos distúrbios metabólicos nos ruminantes (Humer e Zebeli, 2017). Na mais tarefa de fornecer dietas de alto ganho de peso, produtores desprezam o uso de aditivos, ajuste e adaptação dos animais aos novos alimentos, o que predispõe ainda mais desordens metabólicas que podem passar despercebidas (Gonçalves et al., 2001).

Essa diminuição dos níveis de fibra nas dietas tem forçado uma adaptação por mudanças evolutivas no sistema digestório que se tornam preocupantes. Portanto, os conhecimentos sobre morfologia, fisiologia, nutrição e evolução devem ser considerados para obter a devida eficiência em sistemas produtivos modernos (De Tarso et al., 2016). 


\section{Considerações Finais}

$\mathrm{O}$ rúmen funciona como espelho do organismo dos ruminantes, que demonstra através do seu funcionamento, um desencadear de modificações provocadas por diversos fatores. $\mathrm{O}$ conhecimento da fisiologia ruminal em correlação com as enfermidades provindas de distúrbios digestivos passa a ser fundamental para sanidade animal e seu desempenho produtivo.

A busca pelo aumento da produção se interliga totalmente ao bem-estar dos rebanhos e modificações dietéticas em prol desse objetivo provocam um desequilíbrio sistêmico que deve ser levado em consideração. O comportamento do rúmen é um forte indicador da saúde do plantel, e meios de mensuração da avaliação desse compartimento surgem para facilitar o alcance dos objetivos produtivos. Os efeitos da modernização dos sistemas de produção de ruminantes sob a sanidade dos rebanhos podem ser devastadores, o que traz uma necessidade de ferramentas de monitoramento da saúde animal. Portanto, o uso de parâmetros de comportamento ruminal como marcadores da saúde de rebanhos, aparece como uma ferramenta inovadora e promissora diante dos desafios atuais na produção de ruminantes.

\section{Referências}

Albright, J.L. Feeding behavior of dairy cattle. Journal of Dairy Science, 76: 486-498, 1993.

Alexandratos, N.; Bruinsma, J. Food Agriculture Organization of United States Nations. World agriculture towards 2030/2050 the 2012 revision. Disponível em:http://www.fao.org/docrep/016/ap106e/ap1 06e.pdf $>$. Acesso em 10 nov. 2017.

Allen, M.S. Relationship between fermentation acid production in the rumen and the requirement for physically effective fiber. Journal of Dairy Science, 80(7): 144762, 1997.

Almeida, P.E.; Weber, P.S.D.; Burton, J.L.; Zanella, A.J. Depressed DHEA and increased sickness response behaviors in lame dairy cows with inflammatory foot lesions. Domestic Animal Endocrinology, 34: 89-99, 2008.

Ametaj, B.N.; Zebeli, Q.; Iqbal, S. Nutrition, microbiota, and endotoxin-related diseases in dairy cows. Revista Brasileira de Zootecnia, 39: 434-444, 2010a.

Ametaj, B.N.; Zebeli, Q.; Saleem, F.; Psychogios, N.; Lewis, M.J.; Dunn, S.M.; Xia, J.; Wishart, D.S. Metabolomics reveals unhealthy alterations in rumen metabolism with increased proportion of cereal grain in the diet of dairy cows. Metabolomics, 6: 583-594, 2010b.

Beauchemin, K.A. Ingestion and mastication of feed by dairy cattle. Veterinary Clinics North America: Food Animal Practice, 7(2): 439463, 1991.

Bradford, B.J.; Mamedova, L.K.; Minton, J.E.; Drouillard, J.S.; Johnson, B.J. Daily injection of tumor necrosis factor-alpha increases hepatic triglycerides and alters transcript abundance of metabolic genes in lactating dairy cattle. Journal of Nutrition, 139(8): 1451-1456, 2009.

Bradford, B.J.; Yuan, K.; Farney, J.K.; Mamedova, L.K.; Carpenter, A.J. Invited review: inflammation during the transition to lactation: new adventures with an old flame. Journal of Dairy Science, 98: 6631-6650, 2015.

Burfeind, O.; Suthar, V.S.; Voigtsberger, R.; Bonk, S.; Heuwieser, W. Validity of prepartum changes in vaginal and rectal temperature to predict calving in dairy cows. Journal of Dairy Science, 94: 5053-5061, 2011.

Butler, W.R.; Smith R.D. Interrelationships between energy balance and postpartum reproductive function in dairy cattle. Journal of Dairy Science, 72(3): 767-83, 1989.

Calamari, L.; Soriani, N.; Panella, G.; Petrera, F.; Minuti, A; Trevisi, E. Rumination time around calving: An early signal to detect cows at greater risk of disease. Journal of Dairy Science, 97: 3635-3647, 2014.

Catalani, E.; Amadori, M.; Vitali, A.; Bernabucci, U.; Nardone, A.; Lacetera, N. The Hsp72 response in peri-parturient dairy cows: relationships with metabolic and immunological parameters. Cell Stress Chaperones, 15: 781-790, 2010.

Chen, Y.; Oba, M.; Guan, L. Variation of bacterial communities and expression of Toll-like receptor genes in the rumen of steers differing in susceptibility to subacute ruminal acidosis. Veterinary Microbiology, 159: 451-459, 2012.

Clauss, M.; Lechner-Doll, M.; Streich, W. J. Ruminant diversification as an adaptation to the physicomechanical characteristics of forage. A reevaluation of an old debate and a new hypothesis. Oikos, 102: 253-262, 2003.

Constable, P.; Hoffsis, G. F.; Rings, D. M. The reticulorumen: normal and abnormal motor function. The Compendium - Food Animal, 12: 1008-1015, 1990. 
Cooper-Prado, M.J.; Long, N.M.; Wright, E.C.; Goad, C.L.; Wettemann, R.P. Relationship of ruminal temperature with parturition and estrus of beef cows. Journal of Animal Science, 89: 1020-1027, 2011.

De Tarso, S.G.S.; Oliveira, D; Afonso, J.A.B. Ruminants as part of the global food system: how evolutionary adaptations and diversity of the digestive system brought them to the future Br. Journal of Dairy, Veterinary \& Animal Research, 3: 1-7, 2016.

De Tarso, S.G.S. The rumen as a health thermometer: importance of ruminal function to the metabolic balance in ruminants - mini review. Journal of Dairy, Veterinary \& Animal Research, 5: 3-5, 2017.

De Boever, I.L.; Andries, J.I.; De Brabander, D.L.; Cottyn, B.G.; Buysse, X. Chewing activity of ruminants as a measure of physical structure-a review of factors affecting it. Animal Feed Science and Technology, 27(4): 281-291, 1990.

Esposito, G.; Irons, P.C.; Webbb, E.C.; Chapwanya, A. Interactions between negative energy balance, metabolic diseases, uterine health and immune response in transition dairy cows. Animal Reproduction Science, 144:6071, 2014.

FAO/IAEA. Applications of gene-based technologies for improving animal production and health in developing countries. $1^{1 \mathrm{tt}}$ ed. Vienna: Springer Science \& Business Media, 2005. 793p.

Gonçalves, A.L.; Lana, R.L.; Rodrigues, M.T.; Vieira, R.A.M.; Queiroz, A.C.Q.; Henrique, D.S. Padrão Nictemeral do $\mathrm{pH}$ Ruminal e Comportamento Alimentar de Cabras Leiteiras Alimentadas com Dietas Contendo Diferentes Relações Volumoso: Concentrado. Revista Brasileira de Zootecnia, 30(6): 1886-1892, 2001.

Gessner, D.K.; Schlegel, G.; Keller, J.; Schwarz, F.J.; Ringseis, R.; Eder, K. Expression of target genes of nuclear factor E2-related factor 2 in the liver of dairy cows in the transition period and at different stages of lactation. Journal of Dairy Science, 96: 1038-1043, 2013.

Graugnard, D.E.; Moyes, K.M.; Trevisi, E.; Khan, M.J.; Kleisler, D.; Drackley, J.K. Liver lipid content and inflammometabolic indices in peripartal dairy cows are altered in response to prepartal energy intake and postpartal intramammary inflammatory challenge. Journal of Dairy Science, 96: 918-935, 2013.

Hollmann, M.; Miller, I.; Hummel, K.; Sabitzer, S.; Metzler-Zebeli, B.U.; Razzazi-Fazeli, E. et al. Downregulation of Cellular Protective Factors of Rumen Epithelium in Goats Fed High Energy Diet. PLoS ONE, 8: 12, 2013.

Humer, E.; Zebeli, Q. Grains in ruminant feeding and potentials to enhance their nutritive and health value by chemical processing. Animal Feed Science and Technology, 226: 133-151, 2017.

Huzzey; J.M.; Veira, D.M.; Weary, D.M.; Keyserlingk, M.A. Prepartum behavior and dry matter intake identify dairy cows at risk for metritis. Journal of Dairy Science, 90: 32203233, 2007.

Kaufman, E.I.; LeBlanc, S.J.; McBride, B.W.; Duffield, T.F.; DeVries, T.J. Association of rumination time with subclinical ketosis in transition dairy cows. Journal of Dairy Science, 99: 1-15, 2016.

Khafipour, E.; Krause, D.O.; Plaizier, J.C.A. Grain-based subacute ruminal acidosis challenge causes translocation of lipopolysaccharide and triggers inflammation. Journal of Dairy Science, 92: 1060-1070, 2009.

Kovács, L.; Kézér, F.L.; Ruff, F.; Szenc, O. Rumination time and reticulo ruminal temperature as possible predictors of dystocia in dairy cows. Journal of Dairy of Science, 100(2): 1-12, 2017.

Lacetera, N.; Scalia, D.; Bernabucci, U.; Ronchi, B.; Pirazzi, D.; Nardone, A. Lymphocyte functions in overconditioned cows around parturition. Journal Dairy of Science, 88: 2010-2016, 2005.

Leblanc, S.J. Interactions of Metabolism, Inflammation, and Reproductive Tract Health in the Postpartum Period in Dairy Cattle. Reproduction in Domestic Animals, 5(47): 18-30, 2012.

Martz, F.; Belyea, R. Role of particle size and forage quality in digestion and passage by cattle and sheep. Journal of Dairy Science, 69: 1996-2008, 1986.

Oliveira, P.G.; Pires, A.V.; Meyer, P.M.; Susin, I.; Villarreta, E.T.; Rodrigues, P.H.M.; Santos, F.A.P. Gluconeogenic supplements do not affect production, reproductive traits and blood metabolite of holstein cows during the 
transition period. Scientia Agricola, 61(4), 2004

Ospina, P.A.; Nydam, D.V.; Stokol, T.; Overton, T.R. Evaluation of nonesterified fatty acids and $\beta$-hydroxybutyrate in transition dairy cattle in the northeastern United States: critical thresholds for prediction of clinical diseases. Journal of Dairy Science, 93: 546-554, 2010.

Pers-Kamczyc, E.; Zmora, P.; Coeslak, A.; Szumacher-Strabel, M. Development of nucleic acid based techniques and possibilities of their application to rumen microbial ecology research. Journal of Animal and Feed Sciences, 20: 315-337, 2011.

Plaizier, J.C.; Khafipour, E.; Li, S.; Gozho, G.N.; Krause, D.O. Subacute ruminal acidosis (SARA), endotoxins and health consequences. Animal Feed Science and Technology, 172: 9-21, 2012.

Prasad, D.; Pradhan, K. Relative concentration of protozoa, bacteria and some enzymes in the rumen of cattle, buffalo and sheep fed various straw-concentrate diets. Indian Journal of Animal Science, 60: 576-581, 1990.

Regensbogenova M.; Pristas, P.; Javorsky, P.; Moon-van der Staay, S.Y.; Hackstein, J.H.P; Newbold, C.J et al. Assessment of ciliates in the sheep rumen by DGGE. The Society for Applied Microbiology, 39: 144-147. 2004.

Reith, S.; Brandt, H.; Hoy, S. Simultaneous analysis of activity and rumination time, based on collar-mounted sensor technology, of dairy cows over the peri-estrus period. Livestock Science, 170: 219-227, 2014.

Reynolds, C.K.; Aikman, P.C.; Lupoli, B.; Humphries, D.J.; Beever, D.E. Splanchnic metabolism of dairy cows during the transition from late gestation through early lactation. Journal of Dairy Science, 86: 1201-1217, 2003.

Schirmann, K.; Chapinal, N.; Weary, D.M.; Vickers, L.; Kayserlingk, M.A.G. Short communication: rumination and feeding behavior before and after calving in dairy cows.

Journal of Dairy Science, 96: 7088-7092, 2013.

Schröder, U.J.; Staufenbiel, R. Invited review: methods to determine body fat reserves in the dairy cow with special regard to ultrasonographic measurement of backfat thickness. Journal of Dairy Science, 89: 1-14, 2006.
Sievers, A.K.; Kristensen, N.B.; Laue, H.; Wolffram, S. Development of an intraruminal device for data sampling and transmission. Journal of Animal and Feed Sciences, 13: 207-210, 2004.

Soriani, N.; Trevisi, E.; Calamari, L. Relationships between ruminantion time, metabolic conditions, and health status in dairy cows during the transition period. Journal of Animal Science, 90: 4544-4554, 2012.

Soriani, N.; Panella, G.; Calamari, L. Rumination time during the summer season and its relationships with metabolic conditions and milk production. Journal of Dairy Science, 96: 1-13, 2013.

Stangaferro, M.L.; Wijma, R.; Caixeta, L.S.; AlAbri, M.A.; Giordano, J.O. Use of rumination and activity monitoring for the identification of dairy cows with health disorders: Part I. Metabolic and digestive disorders. Journal of Dairy Science, 99(9): 7395-7410, 2016.

Steele, M.A.; Vandervoort, G.; AlZahal, O.; Hook,1 S.E.; Matthews, J.C.; McBride1, B.W. Rumen epithelial adaptation to high-grain diets involves the coordinated regulation of genes involved in cholesterol homeostasis. Physiological Genomics, 43:308-316, 2011a.

Steele, M.A.; Croom J.; Kahler, M.; AlZahal, O.; Hook, S.E. Bovine rumen epithelium undergoes rapid structural adaptations during graininduced subacute ruminal acidosis. American Journal of Physiology: Regulatory, Integrative and Comparative Physiology, 300(6): R1515-R1523, $2011 \mathrm{~b}$.

Suthar, V.S.; Burfeind, O.; Bonk, S.; Dhami, A.J.; Heuwieser, W. Endogenous and exogenous progesterone influence body temperature in dairy cows. Journal of Dairy Science, 95(5): 2381-2389, 2012.

Tewatia, B.S.; Bhatia, S.K. Comparative ruminal biochemical and digestion related physiological characteristics in buffaloes and cattle fed a fibrous diet. Buffalo Journal, 14: 161- 170, 1998.

Urton, G.; Von Keyserlingk, M.A.G.; Weary, D.M. Feeding behavior identifies dairy cows at risk of metritis. Journal of Dairy Science, 88: 28432849, 2005.

Van Soest, P.J. Nutritional ecology of the ruminant. 2nd ed. New York, Cornell University Press, 1994, 476 p. 
Ximenes, L.J.F. Segmento de carnes: não basta ser líder em volume, tem que faturar. Caderno Setorial ETENE, (28): 1-11, 2018

Watanabe, N.; Sakanoue, S.; Kawamura, K.; Kozakai, T. Development of an automatic classification system for eating, ruminating and resting behavior of cattle using an accelerometer. Grassland Science, 54: 231237, 2008.

Weary, D.M.; Huzzey, J.M.; Von Keyserlingk, V.A.G. Using behavior to predict and identify ill health in animals. Journal of Animal Science, 87: 770-777, 2009.
Welch, J.G.; Smith, A.M. Forage quality and rumination time in cattle. Journal of Dairy Science, 53: 797-800, 1970.

Zebeli, Q.; Metzler-Zebeli, B. Interplay between rumen digestive disorders and diet-induced inflammation in dairy cattle. Research in Veterinary Science, 93: 1099-1108, 2012.

Zebeli, Q.; Ghareeb, K.; Humer, E.; MetzlerZebeli, B.U.; Besenfelder, U. Nutrition, rumen health and inflammation in the transition period and their role on overall health and fertility in dairy cows. Research in Veterinary Science, 103: 126-136, 2015. 